OPEN ACCESS

Edited by:

Alexey V. Glukhov,

University of Wisconsin-Madison,

School of Medicine and Public

Health, United States

Reviewed by:

Robert Alan Rose,

University of Calgary, Canada

Francisco J. Alvarado,

University of Wisconsin-Madison,

United States

*Correspondence:

Viacheslav O. Nikolaev

v.nikolaev@uke.de

Specialty section:

This article was submitted to

Cardiac Electrophysiology,

a section of the journal

Frontiers in Physiology

Received: 08 August 2019 Accepted: 31 October 2019

Published: 26 November 2019

Citation:

Dikolayev V, Tuganbekov $T$ and Nikolaev VO (2019) Visualizing Cyclic

Adenosine Monophosphate

in Cardiac Microdomains

Involved in lon Homeostasis.

Front. Physiol. 10:1406.

doi: 10.3389/fphys.2019.01406

\section{Visualizing Cyclic Adenosine Monophosphate in Cardiac Microdomains Involved in Ion Homeostasis}

\author{
Vladimir Dikolayev ${ }^{1,2}$, Turlybek Tuganbekov ${ }^{2}$ and Viacheslav O. Nikolaev ${ }^{1,3 *}$ \\ 'Institute of Experimental Cardiovascular Research, University Medical Center Hamburg-Eppendorf, Hamburg, Germany, \\ ${ }^{2}$ Department of Surgical Diseases, Astana Medical University, Nur-Sultan, Kazakhstan, ${ }^{3}$ German Center for Cardiovascular \\ Research (DZHK), Hamburg, Germany
}

$3^{\prime}, 5^{\prime}$-Cyclic adenosine monophosphate (cAMP) is a key second messenger that regulates function of proteins involved in ion homeostasis and cardiac excitation-contraction coupling. Over the last decade, it has been increasingly appreciated that cAMP conveys its numerous effects by acting in discrete subcellular compartments or "microdomains." In this mini review, we describe how such localized signals can be visualized in living cardiomyocytes to better understand cardiac physiology and disease. Special focus is made on targeted biosensors that can be used to resolve second messenger signals within nanometers of cardiac ion channels and transporters. Potential directions for future research and the translational importance of cAMP compartmentalization are discussed.

Keywords: cyclic adenosine monophosphate, microdomain, imaging, cardiomyocyte, Förster resonance energy transfer biosensor

$3^{\prime}, 5^{\prime}$-Cyclic adenosine monophosphate (cAMP) is a key second messenger that serves, among many ubiquitous functions, as a critical regulator of heart performance and cardiac disease. Stimulation of $\mathrm{G}_{\mathrm{s}}$ protein-coupled receptors on heart muscle cells (cardiomyocytes), including most notably $\beta$-adrenergic receptors ( $\beta$-ARs) stimulated by catecholamines, activates adenylyl cyclase (AC) that catalyzes the generation of cAMP. In turn, cAMP will directly activate protein kinase A (PKA), Epac nucleotide exchange factors for the Rap subfamily of RAS-like small GTPases, cAMP-dependent ion channels, and Popeye domain containing proteins. Classically, cAMP was considered a freely diffusible second messenger. However, in response to adrenergic signaling during physiological fight-or-flight responses, cAMP apparently acts within discrete subcellular microdomains to fine-tune ion channel and transporter activities controlling excitationcontraction coupling. In addition, upon chronic stimulation, compartmentalized cAMP will promote cardiac hypertrophy and remodeling (Fischmeister et al., 2006; Perera and Nikolaev, 2013; Bers et al., 2019). Such microdomains, referred to by some authors as "nanodomains" or "nanocompartments" due to their apparent nanometer scale dimension, are typically organized around scaffolding proteins that bind PKA called A-kinase anchoring proteins (AKAPs). cAMPAKAP compartments have been described for L-type calcium channels (LTCCs) important for calcium influx, ryanodine receptors (RyRs) that mediate release of $\mathrm{Ca}^{2+}$ from intracellular stores, and phospholamban (PLN) that mediates calcium reuptake in diastole via its interaction with the sarcoplasmic/endoplasmic reticulum (SR) calcium ATPase 2a (SERCA2a) pump (Bers, 2002; Lompre et al., 2010; Froese and Nikolaev, 2015). Each of these proteins is part of a multimolecular 
complex containing anchored PKA molecules brought into close proximity to its substrates, specific isoforms of phosphodiesterase (PDE), which catalyze cAMP degradation to both terminate cAMP signaling and provide spatial restriction, as well as other kinases, phosphatases, and other signaling molecules that contribute to local cellular regulation. All these signaling enzymes act together in a highly localized fashion to confer specificity to the diverse physiological and pathophysiological responses triggered by the same second messenger cAMP (Buxton and Brunton, 1983; Mauban et al., 2009; Diviani et al., 2011; Scott et al., 2013). To better understand this type of regulation at the subcellular level and to modulate specific cAMP responses pharmacologically, one needs to gain much deeper insight into local microdomain-specific cAMP dynamics as currently possible only by state-of-the-art live cell imaging techniques.

\section{CYCLIC NUCLEOTIDE BIOSENSORS ENABLE LIVE CELL IMAGING}

The recently rapid development of Förster resonance energy transfer (FRET)-based biosensors has provided researchers with a versatile toolbox for real-time monitoring of cAMP in living cardiac cells (Sprenger and Nikolaev, 2013; Bers et al., 2019; Ghigo and Mika, 2019). cAMP biosensors usually have one of the following five types of design (see Table 1):

1. PKA holoenzyme is composed of two regulatory (R) and two catalytic (C) subunits. The first available FRET biosensor was composed of PKA holoenzyme with fluorescently labeled subunits to monitor its CAMP-dependent dissociation/ re-association (Adams et al., 1991; Zaccolo and Pozzan, 2002).

2. Later, single-chain FRET biosensors were designed based on whole (Zhang et al., 2009; Herbst et al., 2011) or N-terminally truncated (DiPilato et al., 2004; Ponsioen et al., 2004) Epac1 or Epac2 sequence sandwiched between two fluorescent proteins such as enhanced cyan (CFP) and yellow (YFP) fluorescent proteins or their brighter and less $\mathrm{pH}$ sensitive analogues mTurquoise and circularly permuted Venus (Klarenbeek et al., 2015).

3. Fifteen years ago, we introduced a series of much more sensitive and compact CFP-YFP biosensors containing single cAMP binding domains from Epac1, Epac2, PKA, and HCN2 channels (Nikolaev et al., 2004, 2006; Norris et al., 2009). These and the aforementioned sensors have been modified to allow real time cAMP measurements in individual compartments as described below.

4. Recently, Zaccolo and colleagues have introduced a sensor called CUTie (cAMP Universal Tag for imaging experiments), which contains a single cAMP binding domain from PKA RII $\beta$ subunit with YFP inserted into its loop 4-5 and CFP fused to the cAMP binding domain C-terminus (Surdo et al., 2017). This design allows generation of fusion proteins used as targeted biosensors with retained sensitivity and dynamic range. However, this strategy has so far been successful only for N-terminal fusions.

5. Very recently, non-FRET cAMP biosensors called Pink Flamindo and R-FlincA were developed based on the red fluorescent

TABLE 1 | Major types of design and examples of CAMP biosensors.

\begin{tabular}{|c|c|c|c|c|}
\hline Design & Biosensor name & $\begin{array}{l}\text { Dynamic } \\
\text { range }(\%)\end{array}$ & $\mathrm{EC}_{50}(\mu \mathrm{M})$ & References \\
\hline \multirow{9}{*}{$\begin{array}{l}\text { 1. Whole PKA heterotetramer } \\
\text { a. chemically labeled with Fluorophores } \\
\text { b. Fused to CFP and YFP } \\
\text { 2. Whole-length or partially truncated Epac } \\
\text { between CFP/YFP or their mutants }\end{array}$} & & & & \multirow{9}{*}{$\begin{array}{l}\text { Adams et al. (1991) } \\
\text { Zaccolo and Pozzan (2002) } \\
\text { DiPilato et al. (2004); Ponsioen et al. (2004); Zhang et al. } \\
\text { (2009); Herbst et al. (2011); Klarenbeek et al. (2015) }\end{array}$} \\
\hline & FICRhR & 20-30 & 0.09 & \\
\hline & R-CFP;C-YFP & $\sim 20-30$ & $0.5-0.9$ & \\
\hline & CFP-Epac1-YFP & $\sim 15$ & $\sim 50$ & \\
\hline & CFP-Epac2-YFP & $\sim 10-20$ & $\sim 15$ & \\
\hline & CFP-(סDEP, CD)-YFP (H30) & $\sim 50$ & $\sim 10-50$ & \\
\hline & ICUE1/2/3 & $\sim 20-30$ & $\sim 10$ & \\
\hline & Epac-S ${ }^{\mathrm{H} 188}$ & $\sim 100$ & $\sim 49,5$ & \\
\hline & $\begin{array}{l}\text { Epac-S }{ }^{\mathrm{H} 187} \text { (Epac-S }{ }^{\mathrm{H} 188} \text { with } \\
\text { Q270E mutation) }(\mathrm{H} 187)\end{array}$ & $\sim 160$ & $\sim 4$ & \\
\hline \multirow{6}{*}{$\begin{array}{l}\text { 3. Single CNBD sandwiched between YFP and } \\
\text { CFP }\end{array}$} & Epac1-camps & $\sim 30$ & 2.4 & Nikolaev et al. (2004, 2006); Norris et al. (2009); \\
\hline & Epac2-camps & $\sim 20$ & 0.9 & \multirow[t]{5}{*}{ Mukherjee et al. (2016) } \\
\hline & PKA-camps & $\sim 20$ & 1.9 & \\
\hline & HCN2-camps & $\sim 15$ & 6 & \\
\hline & Epac2-camps300 & $\sim 20$ & 0.3 & \\
\hline & mICNBD-FRET & $\sim 30-40$ & 0.07 & \\
\hline $\begin{array}{l}\text { 4. Single CNBD with YFP in the loop } 4-5 \text {, CFP at } \\
\text { C-terminus }\end{array}$ & CUTie & $\sim 20$ & 7.4 & Surdo et al. (2017) \\
\hline \multirow[t]{2}{*}{ 5. Non-FRET } & Pink Flamindo2 & $\sim 400$ & 7.2 & \multirow[t]{2}{*}{ Harada et al. (2017); Ohta et al. (2018) } \\
\hline & R-FlincA & $\sim 600$ & 0.3 & \\
\hline
\end{tabular}

Dynamic range represents a maximally measured \% change in FRET/fluorescence signal in cells or in vitro. EC ${ }_{50}$ shows the affinity of the sensor for cAMP.

CNDB, cyclic nucleotide binding domain. 
protein mApple by either inserting a single cAMP binding domain from Epac1 between amino acids 150 and 151 of mApple or by using a circularly permuted version of this fluorescent protein (cp146) inserted into the PKA regulatory RI $\alpha$ subunit, respectively (Harada et al., 2017; Ohta et al., 2018). No targeted versions of these biosensors have been described so far.

\section{VISUALIZING LOCAL CYCLICAMP IN THE VICINITY OF ION CHANNELS AND TRANSPORTERS USING TARGETED BIOSENSORS}

To monitor cAMP dynamics specifically in various microdomains, several targeted biosensors have been developed. If expressed at appropriate levels, FRET sensors that incorporate full-length PKA or Epac (1 and 2 above) can have a subcellular distribution comparable to that of the endogenous cAMP effector proteins, permitting visualization of varying cAMP levels in multiple PKA and Epac containing compartments across a cell. However, as individual cAMP compartments can be smaller than the resolution of light microscopy, that is, nanometers in diameter, the specific targeting of cAMP sensors to individual compartments is required if cAMP signaling within individual compartments is to be imaged independently of cAMP fluxes that may be quite distinct in neighboring intracellular compartments.

The N-terminal dimerization-docking domain of the PKA regulatory subunit confers AKAP binding. As a first approach to distinguish cAMP compartments, Zaccolo and colleagues fused these sequences derived from the two types of regulatory PKA subunits (RI and RII) to the N-terminus of Epac1-camps to monitor cAMP dynamics at the subcellular sites where endogenous type I and type II PKA are localized. This experimental strategy revealed that PKA type I and II participate in signaling compartments regulated by different PDE isoforms. Type I PKA is more prone to stimulation via prostaglandin and glucagon receptors, whereas type II PKA is predominantly regulated by $\beta$-ARs (Di Benedetto et al., 2008). These compartments seem to mediate distinct functional responses, although it is still not completely understood which particular set of substrates is preferentially phosphorylated by the different PKA holoenzymes. Type II PKA can phosphorylate LTCC, RyR, and PLN, a small protein which regulates the activity of the SERCA2a pump, whereas PKA type I substrates are still unknown or controversial (Di Benedetto et al., 2008). Despite providing powerful biosensors to monitor local cAMP responses, targeting sensors via cAMP effectors has limitations. For example, several AKAPs have been shown to bind each type of PKA regulatory subunits (Diviani et al., 2011), so that each of such sensors can be expected to be simultaneously present at multiple distinct locations in the cell, confounding signals from different compartments when imaged by standard resolution light microscopy. Also, expression of such biosensors would inevitably lead to displacement of endogenous PKA molecules from the microdomains of interest, so that PKA-dependent regulation of local cAMP, PDE, and ion channel activities might be at least partially altered. If possible, these limitations should be considered when designing new targeted biosensors.

To gain further insight into microdomains around calcium handling proteins, our group has developed new versions of the cAMP biosensor Epac1-camps that are localized either to caveolin-rich plasma membrane (Perera et al., 2015) or to major SR membrane proteins SERCA2a and RyR, by fusing the sensor to PLN (Sprenger et al., 2015) or junctin (Berisha et al., 2019), respectively (see Figure 1). These sensors have been expressed in ventricular myocytes of transgenic mice to allow the imaging of cAMP in both normal and disease states. In experiments using myocytes isolated from mice subjected to pressure overload to induce cardiac hypertrophy and early heart failure, these biosensors uncovered a disease driven intracellular redistribution of several PDE families. For example, PDE2 was shown to switch locations between $\beta_{1^{-}}$and $\beta_{2}$-AR-associated plasma membrane microdomains (Perera et al., 2015), while PDE3A switched isoforms from $\mathrm{A} 2$ and $\mathrm{A} 1$ and relocated from the sarcolemma to the SR (Perera et al., 2015; Berisha et al., 2019). This type of PDE redistribution had a functional impact on myocyte contractility, enhancing $\beta_{1}$-AR mediated inotropic increases in contractile force, especially when natriuretic peptide receptors were co-stimulated (Perera et al., 2015). However, microdomain-specific changes in PDE localization apparently occur before a measurable decrease of $\beta_{1}$-AR and PDE3/4 expression at the protein level associated with the onset of ventricular dilation resulting in heart failure with reduced ejection fraction (HFrEF). Therefore, PDE redistribution might contribute to the relative preservation of systolic function during early pathological cardiac remodeling induced by pressure overload, without providing protection from fatal arrhythmia. Arrhythmias in heart failure are also associated with sodium overload. We recently studied healthy and HFrEF rat ventricular myocytes using another newly developed biosensor fused to phospholemman to enable cAMP monitoring in the vicinity of the major cardiac sodium pump, the $\mathrm{Na} / \mathrm{K}$-ATPase (see Figure 1). We found that the phospholemman microdomain is normally regulated by $\beta_{2}$-AR/cAMP and PDE3, while induction of HFrEF by myocardial infarction resulted in a pronounced exchange of PDE3 with PDE2 in this microdomain (Bastug-Özel et al., 2019). The power of targeted biosensors is illustrated by the fact that most of these early pathological alterations in cAMP compartmentation cannot be detected using non-localized, cytosolic sensors or traditional biochemical techniques. Collectively, these studies suggest that each individual microdomain regulating ion homeostasis has a unique local cAMP regulation profile that might change in disease and alter cellular function. An important challenge will be to develop therapeutic strategies to target specifically these microdomains in disease, selectively promoting those cAMP-dependent processes that preserve cardiac function.

Targeted cAMP biosensors have also provided insight into the variation in CAMP signaling induced by stimulation of different cell membrane receptors. For example, in HEK293 cells, cAMP levels monitored by variants of the $\mathrm{H} 30$ biosensor (see Table 1) targeted to the cytosol, plasma membrane, and nucleus were found after prostaglandin receptor stimulation to be relatively high at the plasma membrane, significantly 


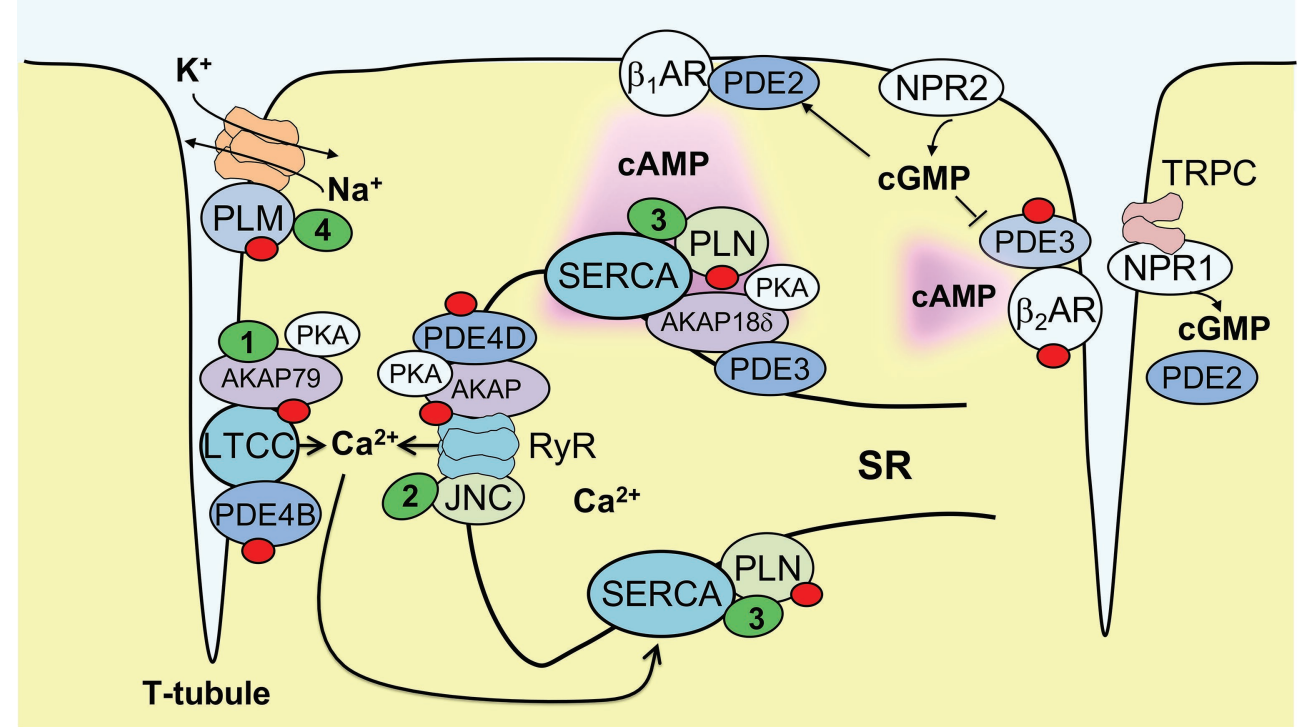

FIGURE 1 | Targeted biosensors used to visualize CAMP in the vicinity of proteins regulating cardiac ion homeostasis. Excitation-contraction coupling in ventricular cardiomyocytes is regulated by the L-type calcium channels (LTCCS) located in membrane invaginations called T-tubules, as well as by sarcoplasmic calcium release (ryanodine receptors, RyR) and reuptake (SERCA pump). Each of these locales forms a microdomain regulated by a specific set of AKAPs and associated PDEs that controls local PKA phosphorylation (marked by red dots). SERCA activity is inhibited by the small regulatory protein phospholamban (PLN); this inhibition is reduced upon PKA mediated PLN phosphorylation. Sodium homeostasis is under tight control by the Na/K-ATPase (NKA) shown in orange. This pump is inhibited by phosholemman (PLM), a regulatory protein that is less active when PKA phosphorylated. cAMP produced after stimulation of $\beta_{1}$-adrenergic receptors ( $\beta$ ARs) diffuses across the cell, activating local pools of PKA in individual microdomains. cGMP can be produced by membrane guanylyl cyclases represented by natriuretic peptide receptors (NPRs) and can either activate PDE2 or inhibit PDE3. NPR1 is confined to T-tubules where it directly interacts with TRPC channels and produces a local pool of cGMP controlled by PDE2. To measure cAMP directly in the vicinities of each ion channel or transporter, targeted FRET biosensors (green) have been developed, such as AKAP79-CUTie (1, for LTCC), Epac1-JNC (2, for RyR), Epac1-PLN or AKAP188-CUTie (3, for SERCA) and PLM-Epac1 (4, for the NKA microdomain). See text for details. JNC - junctin, regulatory protein which forms a stable complex with RyR.

lower in the bulk cytosol and again much higher in the nucleus (Terrin et al., 2006). Likewise, $\beta_{1}$-AR stimulation in adult mouse ventricular cardiomyocytes led to higher cAMP levels detected at SERCA2a using Epac1-PLN sensor as compared to bulk cytosol (Sprenger et al., 2015). Also, cAMP increase measured in the vicinity of AKAP18 $\delta$ (associated with SERCA2a) and AKAP79 (associated with LTCC) imaged using targeted versions of CUTie were higher than in the bulk cytosol (Surdo et al., 2017). Interestingly, this was not the case for a myofilamentassociated microdomain imaged using CUTie fused to troponin I, which showed relatively low cAMP levels at submaximal $\beta$-adrenergic stimulation. This spatial heterogeneity was further accentuated under pathological conditions (Surdo et al., 2017). These studies suggest the existence of a privileged receptor/ microdomain or receptor/calcium handling protein "communication." Mechanistically, this process could be mediated by the concerted action of different PDEs in different compartments (Sprenger et al., 2015). Alternatively, it has been proposed that active $G$ protein-coupled receptors are present on intracellular membranes such as the nuclear envelope or trans-Golgi network where $\beta$-ARs, for example, can be activated by externally applied ligands that gain entry via organic cation transporters and activate cAMP production deep inside the cell (Irannejad et al., 2017). However, this possibility is not yet extensively studied in cardiac cells.

One way to visualize receptor/microdomain communication is to combine targeted biosensors with scanning ion conductance microscopy (SICM). SICM is a non-optical imaging technique which utilizes an electrolyte-filled glass nanopipette as a scanning probe for noncontact visualization of live cell membrane morphology (Korchev et al., 1997). The current flowing through the SICM pipette tip is decreased whenever the tip approaches the cell membrane. By clamping this drop in current at a fixed value, the distance between pipette tip and the membrane can be kept constant, so that scanning the membrane in the X-Y direction can resolve the morphological profile of the membrane with nanometer resolution. SICM as a multimodal imaging technique is useful not only for single cells but also for intact tissue structures (Miragoli et al., 2011). By combining SICM with FRET recordings of cAMP performed during local receptor stimulation via agonist delivery through the scanning pipette, we found that $\beta_{1}$-ARs were diffusely present on the surface and T-tubules of the myocyte plasma membrane, whereas $\beta_{2}$-AR was found exclusively in the T-tubules of healthy rat and mouse 
cardiomyocytes (Nikolaev et al., 2010). Likewise, natriuretic peptide receptors NPR2 and NPR1 show a similar differential distribution pattern on the myocyte plasma membrane resulting in compartmentation of cyclic guanosine monophosphate (cGMP) signaling (Subramanian et al., 2018). Strict confinement of $\beta_{2}$-AR and NPR1 stimulated second messenger signals to T-tubular membrane are mediated by local PDE activities (mostly PDE4 and $\mathrm{PDE} 2$, respectively) and are likely to change in disease due to a redistribution of receptors and PDEs, as shown for $\beta_{2}$-AR and PDE2-4 (Perera et al., 2015; Sprenger et al., 2015). In the future, it would be exciting to combine SICM with targeted cAMP biosensors and with simultaneous ion channel recordings (Sanchez-Alonso et al., 2016) to directly visualize how receptors stimulated at different membrane locations regulate ion channel currents in microdomains. In this case, use of SICM allows activation of spatially resolved membrane structures, such as single T-tubules, with nanometer precision to activate a discrete pool of receptors, without affecting them at other membrane locations. When combined with localized FRET biosensor readouts in certain microdomains inside the cell, this approach provides a possibility to dissect the aforementioned receptor/microdomain communication more directly. It will be exciting to explore whether the same receptor generates different cAMP responses at different subcellular locations and if they are affected by cardiac disease.

\section{FUTURE PERSPECTIVES}

The aforementioned studies have provided an introduction into the microdomains associated with proteins regulating cardiac ion homeostasis. Beyond any doubt, this exciting field of research is still at its very beginning and much deeper insights will follow with time. We propose that future research should include the following topics for investigation:

1. Other differentially targeted biosensors with improved sensitivity and signal-to-noise should be developed and applied to imaging cAMP at ion channels and transporters involved in cardiac function and disease. For excitationcontraction coupling, targets of interest include the Nav1.5 sodium channel, the sodium-calcium exchanger and the potassium channel KCNQ1. All of them can be PKA phosphorylated, and notably KCNQ1 binds an AKAP called Yotiao, whose mutation results in long QT syndrome (Chen et al., 2007). For hypertrophy, an interesting microdomain is likely to be the vicinity of transient receptor potential C (TRPC) channels which is directly associated with NPR1 (Klaiber et al., 2011) (see Figure 1). When designing targeted biosensors, it is important to make sure that they do not perturb the composition and function of cAMP microdomains, for example, due to displacement of endogenous proteins or by altering microdomain regulation. For each newly developed sensor and cells expressing it, a detailed analysis of biochemical and functional properties pertaining to the microdomain of interest should be performed.
2. In vivo mouse models should be developed to dissect the individual contributions of specific PDE, AC, and AKAP families and subfamilies to derive insight into local cAMP signal regulation within individual microdomains. For example, at least three different PDE4 subfamilies (A, B and $\mathrm{D}$ ) and two PDE3 subfamilies (A and B) have been implicated in local cAMP regulation in the cardiac myocyte (Conti and Beavo, 2007; Movsesian et al., 2018). PDE4B is functionally associated with LTCC and PDE4D with RyR (Lehnart et al., 2005; Leroy et al., 2011). Revisiting these studies using targeted biosensors in combination with PDE subfamily specific knockout mice should provide direct microdomain-specific readouts for individual locally acting PDEs and better link them to cardiac function and disease. Most PDE and AKAP global knockout mouse models do not allow the analysis of specific isoform contributions, for example, those of PDE4D3, PDE2A2 or AKAP18ס. There are also no specific isoform-selective pharmacological inhibitors available which could be used for this purpose. Rapid development of CRISPR/Cas9 gene editing technology should be able to overcome this limitation, providing new interesting mouse models or even specific deletions of individual isoforms in primary isolated cardiomyocytes ex vivo. Alternatively, for the PDE4 family in which localization is conferred by alternativelyspliced $\mathrm{N}$-terminal sequences, expression of anchoring disruptor peptides may be particularly useful.

3. The aforementioned small and large animal disease models can be combined with gene therapy approaches aimed at overexpression of microdomain protein components which are downregulated in heart failure such as some PDEs, as it has been done in the pioneering work with SERCA2a (Jessup et al., 2011). Restoration of the expression of individual PDEs may restore normal local cAMP dynamics in functionally relevant microdomains and ameliorate diseaseassociated cardiac dysfunction. Conversely, disruption of PDE-microdomain interactions using specific peptides can be instrumental in augmenting cardioprotective cyclic nucleotide pools, as elegantly shown by Baillie and colleagues for the PDE4D3/Hps20 interaction (Sin et al., 2011). A similar approach is now under evaluation for the SERCA2a/ PDE3A complex. Notably, this latter strategy has been deployed outside the cardiovascular system. For example, expression of the $4 \mathrm{D} 3 \mathrm{~N}$-terminal peptide has been used to disrupt mAKAP $\alpha$-PDE4D3complexes in the retinal ganglion cell to confer neuroprotection (Boczek et al., 2019).

4. It will be important to transit from imaging cardiac myocytes in vitro, whether following culture or immediately ex vivo after isolation from mice, to imaging myocytes in vivo in the living heart. Recently, developed cAMP imaging in isolated Landgendorff perfused hearts that can be combined with electrophysiological recordings (Jungen et al., 2017) or even in open chest in vivo models (Tallini et al., 2006; Frankenreiter et al., 2017) are helping us achieve imaging in a physiological context. Interestingly, cAMP responses and the biochemical composition of signalosomes comprising 
individual compartments can vary depending upon of the anatomical origin of the myocytes, with differences recognized between the atria and base and apex of the ventricles (Wright et al., 2018). These differences should be more carefully considered in the future studies.

5. Last but not least, it will be important to determine whether cyclic nucleotide compartmentation is conserved among mammalian species, including most importantly between rodents and humans. Most research in this field has been performed in rats and mice, given the difficulty in acquiring adult human cardiac myocytes, with the notable exception of a few studies taking advantage of atrial tissue acquired from patients with atrial fibrillation (Molina et al., 2012; Glukhov et al., 2015). Since structure of atrial and ventricular cells and their biochemical makeup including expression of receptors and PDEs are different, additional studies have to be undertaken to better compare mechanisms of cAMP compartmentation in atrial vs. ventricular myocytes. Human studies are of obvious translational importance, and the derivation of cardiac myocytes from human induced pluripotent stem cells (hiPSC-CMs) should ultimately facilitate this research. A current major limitation with using hiPSC-CMs is that current procedures for "maturing" the myocytes yield cells similar to fetal or neonatal myocytes that have significant differences in ultrastructure and excitation-contraction coupling from adult myocytes in vivo, an issue of obvious relevance to the study of compartmentalized signaling. Nevertheless, using a cytosolic cAMP biosensor, clear differences in $\beta$-adrenergic sensitivity and PDE function were detected using hiPSC-CM for patients

\section{REFERENCES}

Adams, S. R., Harootunian, A. T., Buechler, Y. J., Taylor, S. S., and Tsien, R. Y. (1991). Fluorescence ratio imaging of cyclic AMP in single cells. Nature 349, 694-697. doi: 10.1038/349694a0

Bastug-Özel, Z., Wright, P. T., Kraft, A. E., Pavlovic, D., Howie, J., Froese, A., et al. (2019). Heart failure leads to altered beta2-adrenoceptor/cyclic adenosine monophosphate dynamics in the sarcolemmal phospholemman/ $\mathrm{Na}$, K ATPase microdomain. Cardiovasc. Res. 115, 546-555. doi: 10.1093/cvr/cvy221

Berisha, F., Götz, K. R., Wegener, J. W., Jungen, C., Pape, U., Kraft, A. E., et al. (2019). Direct monitoring of cAMP at the cardiac ryanodine receptor using a novel targeted fluorescence biosensor mouse. bioRxiv 623934 [Preprint]. doi: 10.1101/623934 (Accessed October 29, 2019).

Bers, D. M. (2002). Cardiac excitation-contraction coupling. Nature 415, 198-205. doi: $10.1038 / 415198$ a

Bers, D. M., Xiang, Y. K., and Zaccolo, M. (2019). Whole-cell cAMP and PKA activity are epiphenomena, nanodomain signaling matters. Physiology 34, 240-249. doi: 10.1152/physiol.00002.2019

Boczek, T., Cameron, E. G., Yu, W., Xia, X., Shah, S. H., Castillo Chabeco, B., et al. (2019). Regulation of neuronal survival and axon growth by a perinuclear cAMP compartment. J. Neurosci. 39, 5466-5480. doi: 10.1523/ JNEUROSCI.2752-18.2019

Borchert, T., Hübscher, D., Guessoum, C. I., Lam, T. D., Ghadri, J. R., Schellinger, I. N., et al. (2017). Catecholamine-dependent beta-adrenergic signaling in a pluripotent stem cell model of Takotsubo cardiomyopathy. J. Am. Coll. Cardiol. 70, 975-991. doi: 10.1016/j.jacc.2017.06.061

Buxton, I. L., and Brunton, L. L. (1983). Compartments of cyclic AMP and protein kinase in mammalian cardiomyocytes. J. Biol. Chem. 258, 10233-10239

Chen, L., Marquardt, M. L., Tester, D. J., Sampson, K. J., Ackerman, M. J., and Kass, R. S. (2007). Mutation of an A-kinase-anchoring protein causes long- with Takotsubo cardiomyopathy (Borchert et al., 2017). This landmark study should be extended using localized FRET biosensors to better understand microdomain alterations in this and other cardiac diseases. If these alterations are comparable to those happening in adult human cells, hiPSC-CMs could be useful to screening drugs for new cardiovascular therapeutics targeting cyclic nucleotide compartmentation, much as hiPSC-CMs have proven useful for diagnosis and analysis of inherited ion channel diseases such as Long QT syndrome (Sinnecker et al., 2013).

\section{AUTHOR CONTRIBUTIONS}

$\mathrm{VD}, \mathrm{TT}$, and VN discussed the concept, wrote, and edited the manuscript.

\section{FUNDING}

The work in authors' laboratory is supported by the Deutsche Forschungsgemeinschaft (grants NI 1301/3, SFB 1328, and FOR 2060), ERA-Net ERA-CVD, DZHK, and the Gertraud und Heinz Rose-Stiftung. VD was supported by a grant from DAAD.

\section{ACKNOWLEDGMENTS}

We thank Michael S. Kapiloff for critical reading of the manuscript.

QT syndrome. Proc. Natl. Acad. Sci. U. S. A. 104, 20990-20995. doi: 10.1073/ pnas.0710527105

Conti, M., and Beavo, J. (2007). Biochemistry and physiology of cyclic nucleotide phosphodiesterases: essential components in cyclic nucleotide signaling. Annu. Rev. Biochem. 76, 481-511. doi: 10.1146/annurev.biochem.76.060305.150444

Di Benedetto, G., Zoccarato, A., Lissandron, V., Terrin, A., Li, X., Houslay, M. D., et al. (2008). Protein kinase A type I and type II define distinct intracellular signaling compartments. Circ. Res. 103, 836-844. doi: 10.1161/ CIRCRESAHA.108.174813

DiPilato, L. M., Cheng, X., and Zhang, J. (2004). Fluorescent indicators of cAMP and Epac activation reveal differential dynamics of cAMP signaling within discrete subcellular compartments. Proc. Natl. Acad. Sci. U. S. A. 101, 16513-16518. doi: 10.1073/pnas.0405973101

Diviani, D., Dodge-Kafka, K. L., Li, J., and Kapiloff, M. S. (2011). A-kinase anchoring proteins: scaffolding proteins in the heart. Am. J. Physiol. Heart Circ. Physiol. 301, H1742-H1753. doi: 10.1152/ajpheart.00569.2011

Fischmeister, R., Castro, L. R., Abi-Gerges, A., Rochais, F., Jurevicius, J., Leroy, J., et al. (2006). Compartmentation of cyclic nucleotide signaling in the heart: the role of cyclic nucleotide phosphodiesterases. Circ. Res. 99, 816-828. doi: 10.1161/01.RES.0000246118.98832.04

Frankenreiter, S., Bednarczyk, P., Kniess, A., Bork, N. I., Straubinger, J., Koprowski, P., et al. (2017). cGMP-elevating compounds and ischemic conditioning provide cardioprotection against ischemia and reperfusion injury via cardiomyocyte-specific BK channels. Circulation 136, 2337-2355. doi: 10.1161/ CIRCULATIONAHA.117.028723

Froese, A., and Nikolaev, V. O. (2015). Imaging alterations of cardiomyocyte cAMP microdomains in disease. Front. Pharmacol. 6:172. doi: 10.3389/fphar.2015.00172

Ghigo, A., and Mika, D. (2019). cAMP/PKA signaling compartmentalization in cardiomyocytes: lessons from FRET-based biosensors. J. Mol. Cell. Cardiol. 131, 112-121. doi: 10.1016/j.yjmcc.2019.04.020 
Glukhov, A. V., Balycheva, M., Sanchez-Alonso, J. L., Ilkan, Z., Alvarez-Laviada, A., Bhogal, N., et al. (2015). Direct evidence for microdomain-specific localization and remodeling of functional L-type calcium channels in rat and human atrial myocytes. Circulation 132, 2372-2384. doi: 10.1161/ CIRCULATIONAHA.115.018131

Harada, K., Ito, M., Wang, X., Tanaka, M., Wongso, D., Konno, A., et al. (2017). Red fluorescent protein-based cAMP indicator applicable to optogenetics and in vivo imaging. Sci. Rep. 7:7351. doi: 10.1038/s41598-017-07820-6

Herbst, K. J., Coltharp, C., Amzel, L. M., and Zhang, J. (2011). Direct activation of Epac by sulfonylurea is isoform selective. Chem. Biol. 18, 243-251. doi: 10.1016/j.chembiol.2010.12.007

Irannejad, R., Pessino, V., Mika, D., Huang, B., Wedegaertner, P. B., Conti, M., et al. (2017). Functional selectivity of GPCR-directed drug action through location bias. Nat. Chem. Biol. 13, 799-806. doi: 10.1038/nchembio.2389

Jessup, M., Greenberg, B., Mancini, D., Cappola, T., Pauly, D. F., Jaski, B., et al. (2011). Calcium upregulation by percutaneous administration of gene therapy in cardiac disease (CUPID): a phase 2 trial of intracoronary gene therapy of sarcoplasmic reticulum Ca2+-ATPase in patients with advanced heart failure. Circulation 124, 304-313. doi: 10.1161/CIRCULATIONAHA.111.022889

Jungen, C., Scherschel, K., Eickholt, C., Kuklik, P., Klatt, N., Bork, N., et al. (2017). Disruption of cardiac cholinergic neurons enhances susceptibility to ventricular arrhythmias. Nat. Commun. 8:14155. doi: 10.1038/ncomms14155

Klaiber, M., Dankworth, B., Kruse, M., Hartmann, M., Nikolaev, V. O., Yang, R. B., et al. (2011). A cardiac pathway of cyclic GMP-independent signaling of guanylyl cyclase A, the receptor for atrial natriuretic peptide. Proc. Natl. Acad. Sci. U. S. A. 108, 18500-18505. doi: 10.1073/pnas.1103300108

Klarenbeek, J., Goedhart, J., van Batenburg, A., Groenewald, D., and Jalink, K. (2015). Fourth-generation epac-based FRET sensors for cAMP feature exceptional brightness, photostability and dynamic range: characterization of dedicated sensors for FLIM, for ratiometry and with high affinity. PLoS One 10:e0122513. doi: 10.1371/journal.pone.0122513

Korchev, Y. E., Bashford, C. L., Milovanovic, M., Vodyanoy, I., and Lab, M. J. (1997). Scanning ion conductance microscopy of living cells. Biophys. J. 73, 653-658. doi: 10.1016/S0006-3495(97)78100-1

Lehnart, S. E., Wehrens, X. H., Reiken, S., Warrier, S., Belevych, A. E., Harvey, R. D., et al. (2005). Phosphodiesterase 4D deficiency in the ryanodinereceptor complex promotes heart failure and arrhythmias. Cell 123, 25-35. doi: 10.1016/j.cell.2005.07.030

Leroy, J., Richter, W., Mika, D., Castro, L. R., Abi-Gerges, A., Xie, M., et al. (2011). Phosphodiesterase $4 \mathrm{~B}$ in the cardiac L-type $\mathrm{Ca}(2)(+)$ channel complex regulates $\mathrm{Ca}(2)(+)$ current and protects against ventricular arrhythmias in mice. J. Clin. Invest. 121, 2651-2661. doi: 10.1172/JCI44747

Lompre, A. M., Hajjar, R. J., Harding, S. E., Kranias, E. G., Lohse, M. J., and Marks, A. R. (2010). Ca2+ cycling and new therapeutic approaches for heart failure. Circulation 121, 822-830. doi: 10.1161/CIRCULATIONAHA.109.890954

Mauban, J. R., O’Donnell, M., Warrier, S., Manni, S., and Bond, M. (2009). AKAP-scaffolding proteins and regulation of cardiac physiology. Physiology 24, 78-87. doi: 10.1152/physiol.00041.2008

Miragoli, M., Moshkov, A., Novak, P., Shevchuk, A., Nikolaev, V. O., El-Hamamsy, I., et al. (2011). Scanning ion conductance microscopy: a convergent highresolution technology for multi-parametric analysis of living cardiovascular cells. J. R. Soc. Interface 8, 913-925. doi: 10.1098/rsif.2010.0597

Molina, C. E., Leroy, J., Richter, W., Xie, M., Scheitrum, C., Lee, I. O., et al. (2012). Cyclic adenosine monophosphate phosphodiesterase type 4 protects against atrial arrhythmias. J. Am. Coll. Cardiol. 59, 2182-2190. doi: 10.1016/j. jacc.2012.01.060

Movsesian, M., Ahmad, F., and Hirsch, E. (2018). Functions of PDE3 isoforms in cardiac muscle. J. Cardiovasc. Dev. Dis. 5:10. doi: 10.3390/jcdd5010010

Mukherjee, S., Jansen, V., Jikeli, J. F., Hamzeh, H., Alvarez, L., Dombrowski, M., et al. (2016). A novel biosensor to study cAMP dynamics in cilia and flagella. elife 5:e14052. doi: 10.7554/eLife.14052

Nikolaev, V. O., Bünemann, M., Hein, L., Hannawacker, A., and Lohse, M. J. (2004). Novel single chain cAMP sensors for receptor-induced signal propagation. J. Biol. Chem. 279, 37215-37218. doi: 10.1074/jbc.C400302200

Nikolaev, V. O., Bünemann, M., Schmitteckert, E., Lohse, M. J., and Engelhardt, S. (2006). Cyclic AMP imaging in adult cardiac myocytes reveals far-reaching beta 1 -adrenergic but locally confined beta2-adrenergic receptor-mediated signaling. Circ. Res. 99, 1084-1091. doi: 10.1161/01. RES.0000250046.69918.d5
Nikolaev, V. O., Moshkov, A., Lyon, A. R., Miragoli, M., Novak, P., Paur, H., et al. (2010). Beta2-adrenergic receptor redistribution in heart failure changes cAMP compartmentation. Science 327, 1653-1657. doi: 10.1126/ science. 1185988

Norris, R. P., Ratzan, W. J., Freudzon, M., Mehlmann, L. M., Krall, J., Movsesian, M. A., et al. (2009). Cyclic GMP from the surrounding somatic cells regulates cyclic AMP and meiosis in the mouse oocyte. Development 136, 1869-1878. doi: $10.1242 / \mathrm{dev} .035238$

Ohta, Y., Furuta, T., Nagai, T., and Horikawa, K. (2018). Red fluorescent cAMP indicator with increased affinity and expanded dynamic range. Sci. Rep. 8, 1866. doi: 10.1038/s41598-018-20251-1

Perera, R. K., and Nikolaev, V. O. (2013). Compartmentation of cAMP signalling in cardiomyocytes in health and disease. Acta Physiol. 207, 650-662. doi 10.1111/apha.12077

Perera, R. K., Sprenger, J. U., Steinbrecher, J. H., Hubscher, D., Lehnart, S. E., Abesser, M., et al. (2015). Microdomain switch of cGMP-regulated phosphodiesterases leads to ANP-induced augmentation of beta-adrenoceptorstimulated contractility in early cardiac hypertrophy. Circ. Res. 116, 1304-1311. doi: 10.1161/CIRCRESAHA.116.306082

Ponsioen, B., Zhao, J., Riedl, J., Zwartkruis, F., van der Krogt, G., Zaccolo, M., et al. (2004). Detecting cAMP-induced Epac activation by fluorescence resonance energy transfer: Epac as a novel cAMP indicator. EMBO Rep. 5, 1176-1180. doi: 10.1038/sj.embor.7400290

Sanchez-Alonso, J. L., Bhargava, A., O’Hara, T., Glukhov, A. V., Schobesberger, S., Bhogal, N., et al. (2016). Microdomain-specific modulation of L-type calcium channels leads to triggered ventricular arrhythmia in heart failure. Circ. Res. 119, 944-955. doi: 10.1161/CIRCRESAHA.116.308698

Scott, J. D., Dessauer, C. W., and Tasken, K. (2013). Creating order from chaos: cellular regulation by kinase anchoring. Annu. Rev. Pharmacol. Toxicol. 53 , 187-210. doi: 10.1146/annurev-pharmtox-011112-140204

Sin, Y. Y., Edwards, H. V., Li, X., Day, J. P., Christian, F., Dunlop, A. J., et al. (2011). Disruption of the cyclic AMP phosphodiesterase-4 (PDE4)HSP20 complex attenuates the beta-agonist induced hypertrophic response in cardiac myocytes. J. Mol. Cell. Cardiol. 50, 872-883. doi: 10.1016/j. yjmcc.2011.02.006

Sinnecker, D., Goedel, A., Laugwitz, K. L., and Moretti, A. (2013). Induced pluripotent stem cell-derived cardiomyocytes: a versatile tool for arrhythmia research. Circ. Res. 112, 961-968. doi: 10.1161/CIRCRESAHA.112.268623

Sprenger, J. U., and Nikolaev, V. O. (2013). Biophysical techniques for detection of cAMP and cGMP in living cells. Int. J. Mol. Sci. 14, 8025-8046. doi: 10.3390/ijms 14048025

Sprenger, J. U., Perera, R. K., Steinbrecher, J. H., Lehnart, S. E., Maier, L. S., Hasenfuss, G., et al. (2015). In vivo model with targeted cAMP biosensor reveals changes in receptor-microdomain communication in cardiac disease. Nat. Commun. 6:6965. doi: 10.1038/ncomms7965

Subramanian, H., Froese, A., Jonsson, P., Schmidt, H., Gorelik, J., and Nikolaev, V. O. (2018). Distinct submembrane localisation compartmentalises cardiac NPR1 and NPR2 signalling to cGMP. Nat. Commun. 9:2446. doi: 10.1038/ s41467-018-04891-5

Surdo, N. C., Berrera, M., Koschinski, A., Brescia, M., Machado, M. R., Carr, C., et al. (2017). FRET biosensor uncovers cAMP nano-domains at betaadrenergic targets that dictate precise tuning of cardiac contractility. Nat. Commun. 8:15031. doi: 10.1038/ncomms15031

Tallini, Y. N., Ohkura, M., Choi, B. R., Ji, G., Imoto, K., Doran, R., et al. (2006). Imaging cellular signals in the heart in vivo: cardiac expression of the high-signal Ca2+ indicator GCaMP2. Proc. Natl. Acad. Sci. U. S. A. 103, 4753-4758. doi: 10.1073/pnas.0509378103

Terrin, A., Di Benedetto, G., Pertegato, V., Cheung, Y. F., Baillie, G., Lynch, M. J., et al. (2006). PGE(1) stimulation of HEK293 cells generates multiple contiguous domains with different [cAMP]: role of compartmentalized phosphodiesterases. J. Cell Biol. 175, 441-451. doi: 10.1083/jcb.200605050

Wright, P. T., Bhogal, N. K., Diakonov, I., Pannell, L. M. K., Perera, R. K., Bork, N. I., et al. (2018). Cardiomyocyte membrane structure and cAMP compartmentation produce anatomical variation in beta2AR-cAMP responsiveness in murine hearts. Cell Rep. 23, 459-469. doi: 10.1016/j. celrep.2018.03.053

Zaccolo, M., and Pozzan, T. (2002). Discrete microdomains with high concentration of cAMP in stimulated rat neonatal cardiac myocytes. Science 295, 1711-1715 doi: $10.1126 /$ science. 1069982 
Zhang, C. L., Katoh, M., Shibasaki, T., Minami, K., Sunaga, Y., Takahashi, H., et al. (2009). The cAMP sensor Epac2 is a direct target of antidiabetic sulfonylurea drugs. Science 325, 607-610. doi: 10.1126/science.1172256

Conflict of Interest: The authors declare that the research was conducted in the absence of any commercial or financial relationships that could be construed as a potential conflict of interest.
Copyright (c) 2019 Dikolayev, Tuganbekov and Nikolaev. This is an open-access article distributed under the terms of the Creative Commons Attribution License (CC BY). The use, distribution or reproduction in other forums is permitted, provided the original author(s) and the copyright owner(s) are credited and that the original publication in this journal is cited, in accordance with accepted academic practice. No use, distribution or reproduction is permitted which does not comply with these terms. 